\title{
Exchange of high and low energy photons in ultraperipheral relativistic heavy-ion collisions
}

\author{
G.Baur ${ }^{\mathrm{a}}$

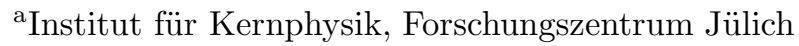 \\ D-52428 Jülich, Germany
}

Ultraperipheral collisions at collider energies are a useful tool to study photon-hadron (proton/nucleus) and photon-photon interactions in a hitherto unexplored energy regime. Theoretical tools to study these processes are briefly described. Some current results and problems are discussed.

\section{Introduction}

Hadron-hadron collisions can be an interesting tool to study photon-photon and photonhadron interactions. Of course, hadron-hadron collisions are dominated by strong interactions between the hadrons. However, by choosing collisions with large impact parameter b (or, equivalently, small momentum transfer) one can suppress these strong interactions (so-called ultraperipheral collions (UPC)). An early observation of dimuon pairs at the ISR without the production of hadrons is described by F.Vannucci [1]. Dielectrons from ultraperipheral peripheral interactions at the Tevatron were discussed at this workshop [2].

A fast moving charged particle acts like a spectrum of quasireal photons. This equivalent photon spectrum is soft, with a maximum photon energy increasing with beam energy. In heavy ion collisions this field is so strong that there is multiple photon exchange and cross sections, especially for soft processes, can be huge.

In Section 2 the characteristic properties of the equivalent photon spectrum are recalled. Glauber or semiclassical models are used to treat multiphoton processes. Vector meson production at RHIC energies is discussed in Section 3. An especially interesting feature is the transverse momentum distribution. Section 4 is devoted to the special problem of $e^{+} e^{-}$pair production. A very brief outlook on prospects at the LHC is given in Section 5, conclusions are given in Sec- tion 6 . There are various reviews which reflect the progress of the field during the last decades 34516789910.

\section{Theoretical description of UPC}

\subsection{One photon exchange, equivalent pho- ton aproximation}

The time-dependent electromagnetic field of a fast moving charged particle can be thought of as a spectrum of (quasireal, or equivalent) photons 11, see Figure 1. The determination of the equivalent (or Weizsäcker-Williams) photon spectrum corresponding to a fast particle moving past an observer on a straight line path with impact parameter $b$ is a textbook example [12].

The probability $P(b)$ of a specific photonhadron reaction to occur in a collision with an impact parameter $b$ is given by $P(b)=$ $N(\omega, b) \sigma_{\gamma h}(\omega)$, where $\sigma_{\gamma h}$ is the corresponding photoproduction cross section. The equivalent photon spectrum can be calculated analytically, a useful approximation for qualitative considerations is

$N(\omega, b)=\frac{Z^{2} \alpha}{\pi^{2} b^{2}}$

for $\omega<\frac{\gamma}{b}$ and zero otherwise. The nuclear charge is given by $Z$, heavy ions have particularly high photon fluxes, however, this is partially offset by the lower ion-ion luminosities, as compared to the p-p case.

The impact parameter $\mathrm{b}$ is restricted to

$b>b_{\text {min }} \sim R_{1}+R_{2}$ 


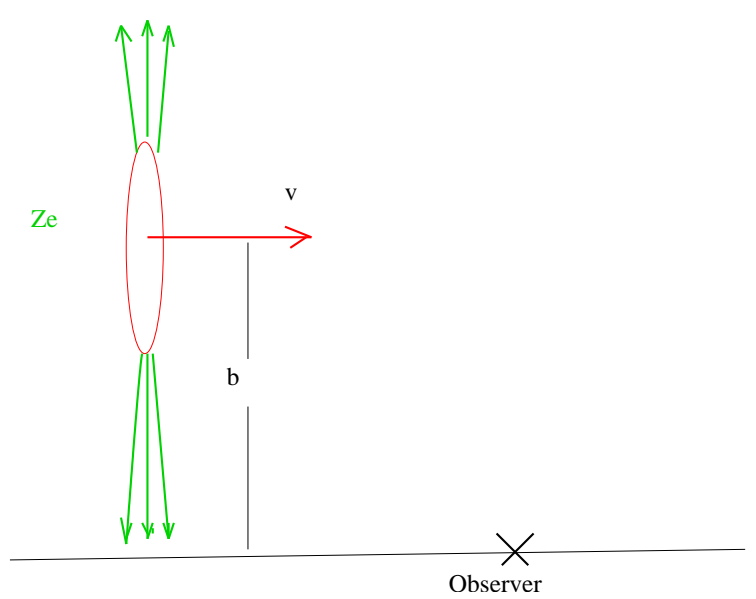

Figure 1. A fast charged particle moving on a straight line with impact parameter b causes a time-dependent electromagnetic field at the point of the observer. This field corresponds to a spectrum of equivalent photons.

where $R_{1}$ and $R_{2}$ denote the sizes of the hadrons.

For heavy ion scattering the Coulomb parameter $\eta \equiv \frac{Z^{2} e^{2}}{\hbar v} \sim Z^{2} / 137$ is much larger than unity and it is in principle possible to determine the impact parameter by measuring the angle of Coulomb scattering. Whereas this is experimentally feasible at lower $(\sim \mathrm{GeV} / \mathrm{A})$ energies [13, this angle is too small at collider energies. So one generally measures quantities integrated over all impact parameters. Too small impact parameters are recognized since the event is dominated by the violent strong interactions.

The photon spectrum Eq. 1 extends up to a maximum photon energy given by

$\omega_{\max }=\frac{\gamma}{b_{\min }}$.

This energy is about $3 \mathrm{GeV}$ at RHIC (Au-Au, $\gamma \sim 100$ ), and $100 \mathrm{GeV}$ at LHC (Pb-Pb, $\gamma \sim$ 3000 ) in the collider system. For p-p collisions at the LHC it is several hundreds of GeV. With the possibitity to tag on these photons by observing the forward protons this is of great interest, as was discussed at this workshop.

\subsection{Improvement on equivalent photon approximation}

As the name implies, the equivalent photon aproximation is an approximation. The virtual, spacelike photon which is exchanged is assumed to be real, and a suitable cut-off has to be introduced. This cut-off parameter enters only in a logarithm, and it is not sensitive to details. It is of course also possible to calculate the one-photon exchange process in a direct way by evaluating the corresponding Feynman graph, where the exchanged photon is treated as a virtual particle. It is straightforward to introduce electromagnetic formfactors for the protons or nuclei. They are suffiently well known for the present purposes. This could be directly implemented into computer programs like Madgraph (see Michel Herquert, this workshop). The approximations introduced in the equivalent photon approximation are discussed in detail in [14, see also [15].

\subsection{Multiphoton processes, Glauber ap- proximation}

For heavy ions the probability of an electromagnetic interaction in ultraperipheral collisions is especially large, and multiphoton processes occur, see e.g. 16. We mention $e^{+} e^{-}$pair production where the impact parameter dependent total pair production probability $P(b)$ is of order unity. Multiple pairs can be produced, however they may be hard to detect due to their low transverse momentum. The nuclear giant dipole resonance is excited with probabilities of the order of one third. For a reliable estimate of the giant dipole excitation probability on can use the global formula

$P(b)=5.4 \times 10^{-5} Z^{3} N A^{-2 / 3} f m^{2}$

where a collision of two identical nuclei with mass number $\mathrm{A}$ and neutron number $\mathrm{N}$ was assumed. It is based on the Thomas-Reiche-Kuhn sum rule, and the excitation energy of the giant dipole resonance is assumed to be $80 A^{-1 / 3} \mathrm{MeV}$. For more accurate numbers one uses the experimentally known photonuclear cross sections and folds them with the equivalent photon spectrum. In Figure 2 one of the graphs is shown which leads to the electromagnetic production of a $\rho^{0}$ along with the 
excitation of the giant dipole resonance. These graphs can conveniently be evaluated in semiclassical or eikonal theories [16]. Whereas the total cross section for one-photon exchange processes depends only logarithmically on the minimum cut-off radius $\mathrm{R}$, the $\mathrm{N}$-photon exchange process scales as $1 / R^{2(N-1)}$. Thus it becomes more important to have a reliable value for this strong cut-off parameter in the case of multiphoton exchange.

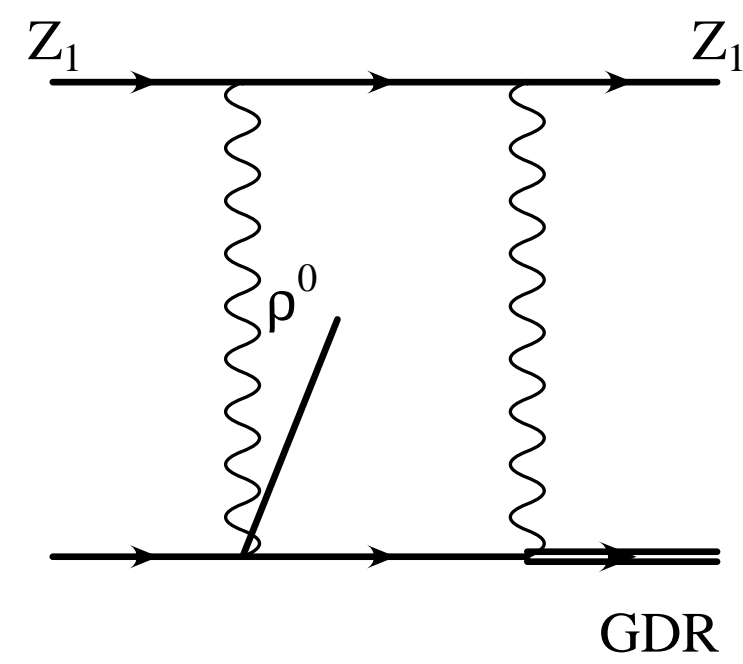

Figure 2. A graph contributing to the simultaneous production of a $\rho$-meson and the excitation of the giant dipole resonance (GDR).

The giant dipole resonance decays dominantly into a neutron and a residual nucleus. The neutron can be detected in the forward direction. This can serve as a luminosity monitor and also as a trigger on UPC [17].

\section{Vector meson production at RHIC}

Recently $\rho^{0}$ photoproduction in ultraperipheral relativistic heavy ion collisions at $\sqrt{s_{N N}}=200$ $\mathrm{GeV}$ was reported by the STAR collaboration [18. Two kinds of triggers were used, a topology trigger and a trigger based on the observation of neutrons in the Zero Degree Calorimeter (ZDC). In the latter case, the vector meson photoproduction is accompanied by mutual electromagnetic excitation of the giant dipole resonance. I would like to point out that a reliable theoretical value for the giant resonance excitation probability (or rather photoneutron cross section) has to be used in order to extract absolute values for the differential photoproduction cross section.

A feature unique to photoproduction in hadron-hadron collisions is an interference effect [19]: a vector meson can be produced by a photon originating from either of the hadrons. It was shown in [19 that this interference effect leads to a reduction of the transverse momentum spectrum of the vector mesons for small transverse momenta. Another theoretical approach [20] leads to very similar conclusions. In this paper Glauber and semiclassical methods (shown to be essentially equivalent) are used to describe the interference phenomenon. The differential cross section is found to be

$\frac{d^{3} \sigma}{d^{2} v_{\perp} d Y}=\frac{1}{2(2 \pi)^{3}} \sum_{e_{V}} \int|a|^{2} d^{2} b$

where

$a=a_{1}(b) a_{2}(b)\left(a_{V}\left(\vec{b}, \vec{v}_{\perp}, Y\right)+e^{-i \vec{v}_{\perp} \cdot \vec{b}} a_{V}\left(-\vec{b}, \vec{v}_{\perp},-Y\right)\right)$

Here $a_{1}$ and $a_{2}$ denote the nuclear excitation, the amplitude for vector meson photoproduction is denoted by $a_{V}$. The vector meson perpendicular momentum is $\vec{v}_{\perp}$, Y is the rapitidy and $\vec{b}$ the impact parameter. (Preliminary) experimental results from STAR/RHIC indeed show a dip for small transverse momenta, see e.g. Ref. [10].

\section{Electron-positron pair production}

Electron-positron pair production in ultrarelativistic heavy ion collisions was recently reviewed in [21. The electromagnetic fields are very strong, of the order of or even larger than the Schwinger critical field strength which is given by

$E_{c}=\frac{m_{e}^{2} c^{3}}{e \hbar} \simeq 1.3 \times 10^{16} \mathrm{~V} / \mathrm{cm}$

where $m_{e}$ denotes the electron mass. However, these fields act only for a very short time and per- 
turbation theory is an appropriate tool to study these processes. Higher order effects are present and were studied theoretically over the past years, and the effects are well understood. For further details and a comparison to the physics of ultraintense laser pulses we refer to [21.

Electron-positron pair production in ultraperipheral collisions accompanied by mutual giant dipole excitation was measured at STAR and analysed with equivalent photon method [22]. A lowest order QED (two-photon pair production) calculation is given in 23. In view of the limited statistics, agreement between theory and experiment can be considered as satisfactory.

In a more recent analysis by A. Baltz 24] the neutron production probability is treated in more detail and higher order QED effects are included. The author concludes that the experiment 22] is in good agreement with the higher order calculation.

\subsection{Bound-free pair production}

In bound-free pair production the electron is produced in a bound atomic orbit (K-,L-,..shell). It scales approximately as

$\sigma \sim \frac{Z^{7} \ln \gamma \delta_{l 0}}{n^{3}}$

where $\mathrm{n}$ and $\mathrm{l}$ denote the principal and agular momentum quantum numbers of the atomic bound state. These ions with their changed charge-to mass ratio will get lost from the beam and they will heat up the beam pipe in a hot spot. It was identified as a serious limit for the luminosity in $\mathrm{Pb}-\mathrm{Pb}$ collisions at LHC. The bound-free pair production cross section was recently measured at RHIC [25]. Agreement with theory [26] is good. The bound-free pair production mechanism was also used in the production of fast antihydrogen at LEAR in 1996 by W. Oelert et al. 27].

\section{Opportunities for UPC at LHC}

The events caused by ultraperipheral collisions may be called 'silent events', as compared to the voilent central collisions. However, the collision of a $100 \mathrm{GeV}$ equivalent photon with another one of similar energy can lead to lead to events that are not so 'silent'. Anyway, the events are there, but experimentalists must find ways to trigger on them.

The maximum photon energy scales linearly with the Lorentz factor $\gamma$, see eq. 3. This leads to a significant widening of the opportunities at LHC as compared to RHIC. A most promising area is low-x QCD studies. The experiments at HERA have shown that photoproduction processes provide a well-understood probe of the gluon density in the proton. At LHC, such processes could be extended to invariant $\gamma p$ energies exceeding the maximal HERA energy by a factor of 10. This would allow to use dijet (charm, etc.) production to measure the gluon density in the proton and/or nucleus down to $x \sim 3 \times 10^{-5}$. Ultraperipheral collisions would also allow one to study the coherent production of heavy quarkonia, $\gamma+A \rightarrow J / \Psi(\Upsilon)+A$ at $x \lesssim 10^{-2}$, and to investigate the propagation of small dipoles through the nuclear medium at high energies, see Ref. [28, see also Refs. 29130. Dijet production via photon-gluon fusion is calculated in Ref. 31. Very large rates are obtained that will considerably extend the HERA $x$ range.

In addition to diffractive processes in protonproton collisions at LHC also a rich program of proton-photon and photon-photon physics can be pursued, see Ref. 32. The photon flux is lower as compared to the heavy ion case due to the $Z^{2}$ factor, but this is at least partly compensated by higher beam luminosities. The photon spectrum is harder due to the smaller size as compared to the heavy ions, this leads to a lower value of $b_{\min }$ in Eq. 3. Possibilities for electroweak physics and beyond were discussed at the present workshop. Tagging on photon energy by measuring the energy loss of the scattered protons in the forward detector TOTEM is an important feature.

The production of the Higgs boson by photonphoton fusion would be of obvious interest. A value of $3.9 \mathrm{nb}$ for $\mathrm{Au}-\mathrm{Au}$ collisions at the $\mathrm{LHC}$ was given recently in 33. This is a large value, as compared to numbers reported previously, see e.g. Ch. 7.2 .7 of [6]. Absorption seems to be treated somewhat differently in 33 as compared to the methods developed in 34 35]. 


\section{Conclusions}

Ultraperipheral collisions at the LHC can provide a new means of studying small $x$ QCD, electroweak physics and physics beyond the standard model. Some issues related to the theoretical description of ultraperipheral heavy ion collisions are briefly discussed in this contribution.

\section{Acknowledgments}

I would like to thank David d'Enterria, Michael Klasen and Krzystof Piotrzkowski for their kind invitation to this timely and stimulating workshop. I am grateful to Carlos Bertulani, Kai Hencken, and Dirk Trautmann for their collaboration over many years. I also wish to thank Spencer Klein and Joakim Nystrand for discussions.

\section{REFERENCES}

1. F.Vannucci, in G.Cochard (Ed), $\gamma \gamma$ Collisions, Proceedings, Amiens 1980, Lecture Notes in Physics, Vol. 134, Springer, Heideoberg, Berlin, New York, 1980, p. 238

2. J.Pinfold, these proceedings

3. C. A. Bertulani, and G. Baur, Phys. Rept. 163299 (1988)

4. F. Krauss, M. Greiner, G. Soff, Prog. Part. Nucl. Phys. 39503 (1997)

5. G. Baur, K. Hencken, D. Trautmann, S. Sadovsky, Y. Kharlov, Phys. Rept. 364359 (2002)

6. Heavy ion physics programme in CMS G. Baur et al. Eur. Phys. J. C32 s69 (2003)

7. Carlos A. Bertulani, Spencer R. Klein, and Joakim Nystrand, Annual Review of Nuclear and Particle Science 55271 (2005)

8. A. Baltz et al., Mini-proceedings of the ECT* workshop on 'Photoproduction at collider energies: from RHIC and HERA to the LHC, arXiv: hep-ph/0702212

9. G.Baur in PHOTON 2007, Paris, 9-13 July 2007 Proceedings to be published in Nucl. Phys. B, arXiv:0711.2882

10. A. Baltz et al., Phys. Rept. 4581 (2008), arXiv:0706.3356

11. E. Fermi, Z.Physik 29315 (1924)
12. J.D. Jackson, Classical Electrodynamics (Wiley, New York,1975)

13. T. Aumann, Eur. Phys. J. A26 441 (2005)

14. V. M. Budnev, I. F. Ginzburg, G. V. Meledin, V. G. Serbo Phys. Rept. 15181 (1975)

15. F.Halzen and A.D. Martin, Quarks and leptons: an introductory course in modern particle physics, New York, Wiley 1984

16. G. Baur, K. Hencken, A. Aste, D. Trautmann , S. R. Klein, Nucl. Phys. A729 787 (2003)

17. M. Chiu et al. Phys. Rev. Lett. 89012302 (2002)

18. B. I. Abelev et al. Phys. Rev. C77 034910 (2008)

19. Spencer R. Klein and Joakim Nystrand Phys. Rev. Lett., 842330 (2000)

20. K. Hencken, G. Baur, and D. Trautmann, Phys. Rev. Lett. 96012303 (2006)

21. G. Baur, K. Hencken, D. Trautmann, Phys. Rept. 4531 (2007)

22. J. Adams et al. Phys. Rev. C70 031902R (2004)

23. K. Hencken, G. Baur, D. Trautmann, Phys. Rev. C69 054902(2004)

24. A. Baltz, Phys. Rev. Lett. 100062302 (2008)

25. R. Bruce et al., Phys. Rev. Lett. 99144801 (2007)

26. H. Meier et al., Phys. Rev. A63 032713 (2001)

27. G. Baur et al., Phys. Lett. B 368251 (1996

28. Leonid Frankfurt, Mark Strikman, and Christian Weiss Annual Review of Nuclear and Particle Science 55403 (2005)

29. Yuri Ivanov, Boris Kopeliovich, and Ivan Schmidt, arXiv:0706.1532

30. V. P. Gonçalves and M. V. T. Machado, arXiv:0706.2810

31. Mark Strikman, Ramona Vogt, Sebastian White Phys. Rev. Lett. 96082001 (2006)

32. Prospects for Diffractive and Forward Physics at the LHC CERN/LHCC 2006-039/G-124

33. E. Levin and J. Miller, arXiv:0801.3593

34. G. Baur, L. G. Ferreira Filho, Nucl. Phys. A518 786 (1990)

35. N. Cahn, J. D. Jackson, Phys. Rev. D42 3690 (1990) 